\title{
Active Exoskeleton Hand Exerciser Using Multisensory Feed Back
}

\author{
Arpan Bhattacharyya, Arindam Dey Sarkar and Sahadev Roy
}

\begin{abstract}
Nowadays the leading cause of disability is stroke. The main reasons of cerebra vascular accident counted as Ischemia or Haemorrhage in the brain which ultimately results in stroke. It is widely agreed that natural recovery as well as functional recovery of the body takes place in the first month of the incident of the stroke victim. For a better improvement some organisation for neuroscience has need to be put in order. Neural pathways which are damaged earlier can be reformed with neurones present in activity and also can be engaged. The exoskeleton hand exerciser may be used for guidance or training device for patients following stroke to vigorously train their affected hand functions in normal condition. For different kind of disabilities there are different actuation and control techniques. Exoskeleton hand exerciser is a prosthetics device for paralyzed patients who have lost their control over hand. It is very useful thing by which they can easily do their works. Not only for the paralyzed people it is now very useful for military or forces. It can easily lift the heavy weighs of different weapons. This paper also explores the art of the exoskeleton exerciser for the palm.
\end{abstract}

\section{Keywords}

Exercisers for paralyzed patient; Exoskeleton hand exerciser; Rehabilitation exerciser; Upper arm rehabilitation exoskeletons.

\section{INTRODUCTION}

Rehabilitation Robotics for the first time introduced in North America. The first rehabilitation robot was consisted of floor mounted and 4 degree of freedom exoskeleton was developed in 1960's. In the early 1978 researchers started to find the method of creating exoskeletons [1]. It progressed from the science fable stuff to nearly market products. Many challenges came into account while developing Exoskeleton. For a mechanical suit the first patent appeared in 1980, but exoskeleton which is both real and imagine recently adopted. In the late 1960s, human exoskeleton device is powered by the patent and the science fiction research started parallel with research organization in US and the former country

Authors Details:

*Arpan Bhattacharyya

Dept. of CSE, Government College of Engineering and Textile Technology, Berhampore, India

e-mail: arpanbhattacharyya60@gmail.com

Arindam Dey Sarkar

Dept. of ECE, National Institute of Technology, Arunachal Pradesh, India

e-mail: deysarkar1991@gmail.com

Sahadev Roy

Dept. of ECE, National Institute of Technology, Arunachal Pradesh, India

e-mail:sdr.ece@nitap.in

Editor: Subject Category: Mechatronics

Sub Category: Intelligent System
Yugoslavia. On the other hand, the former was mainly focused to upgrade the technologies to increase the ability of an ablebodied human, often for defense purposes, while latter it was developing for the disabled persons or physically challenged peoples [2].

Now a day the leading cause of disability is stroke. The main reasons of cerebra vascular accident counted as Ischemia or Hemorrhage in the brain which ultimately results in stroke [3]. Fortunately over 75 percent of the patients survive but residual disabilities attacks majority of them with up to one third suffering from intense disabilities predominantly on the shoulder and the hand [4]. Hemiplegic, the most frequent physical, cognitive effects result from brain stroke [5]. Traumatic injury as well as arthritis or regional pains are the main causes of a patient's disability. For the case of brain stroke victims, it is observed that natural recovery as well as functional reinstallation of the body takes place in the very first month of the stroke victim after the incident. For a better improvement some organization for neuroscience has need to be put in order [6]. Neural pathways which are damaged earlier can be reformed with neurons present in activity and also can be engaged. Restoration is very much important to fix flexor muscle injuries or to remove adhesion following operation. Physiotherapists manually performed the reclamation task. But people faces problem in terms of personnel cost and lack in their motivation to do the exercises. An uninterrupted motion provided by some devices helps physiotherapist to apply it on patient's hand. After arm operations it's necessary to go through reclamation to restore previous sleight. It has brutal effects on socio economic field if rehabilitation does not succeed. The lack of measurements in reproducibility is the other difficulty rises during healing process. These are desired to determine the restriction in the sleight of the hand and to assess the treatment progression. For successful hand restoration; we need two parameters which are very important that is to identify impairment and monitoring the progress of the systems. In this paper, we retrospect the history and discuss the capability of exoskeleton hand. The design overviews which are include hardware, actuator, sensor, and control system of the devices. In this paper, the role of exoskeleton hand exercisers for paralyzed 
patient is discussed briefly in Section II. Overview of the controller section is discussed in Section III. Mechatronics model of the exoskeleton is analyzed in the Section IV.

\section{THE ROLE OF EXOSKELETON HAND EXERCISERS FOR PARALYZED PATIENT}

Research on exoskeletons hand devices is still interesting and going on in different R\&D center. Hand exoskeletons design and developed for other applications will be considered as well as. The main applications of the devices fit interaction along with a virtual fact or manipulations done remotely with robot arms [7]. Since most of the exoskeleton are like as master arm have a similar type kinematics design to that of the slave arm, with actuator controlled by bulky and heavy electric motors. Many researcher developed force reflecting master arm and use various type of modern actuators their motive is to make compact and lightweight exoskeleton by providing sufficient space and force reflection capabilities by reducing degree of freedom [8]. Jamshed Iqbal et.al proposed Hand Exoskeleton System (HEXOSYS) by using multi objective optimization or reformation [9] technique by receiving inspiration from the human hand. The optimization or reformation algorithms select the most beneficial part of isotropy, dexterity and application of perpendicular forces on the finger digits. Moreover, a multi experiments on the human hand by means of adequate sensory instrumentation directed to arrange of actuators which lead to a rehabilitation device that is coherent with the human hands force capabilities. The supply of force on top of position feedback gives the therapist a quantitative feedback and would mean a better organized rehabilitation process [10]. The methods used for actuation were planned so that it would mimic natural motions the maximum amount possible. The ultimate design was thought of in terms of its combination of movements, controllability along with the placement of components. The anatomy of the human hand was closely studied.

Some researchers use the tactile sensation and manipulation to develop intelligent exerciser. The grip force may be control by vision-based slip margin estimation by its direct feedback the mechanical. Some exerciser has 4 fingers with 3 DOF. Its particularly designed by using gear mechanism and also has all three actuators that can be used in the palm without making use of wire-driven mechanism, i.e. it has stress-free the limitation on the space of movement of actuators. A tactile fingertip sensor which is vision-based is also presented. It consists of an embedded small camera, a transparent semi spherical gel, and a force sensor use to implement to reduce direct slip. It is tricky to raise the spatial resolution since several strain gauges must be placed within the sensor. To solve the problems mentioned above, a visionbased tactile sensation and grip-force control method has been proposed. The method proposed has the following advantages, a stable grip force control by a direct feedback control mechanisms to meet the slip margin and a highly accurate vision-based area of contact measurement. The area in contact was calculated outside from the fingertip. In this paper, they represent a newly developed fingertip sensor for the exoskeleton hand with normal force increased to the power of $1 / 3$ [11]. Once the entire contact surface slips, the elastic object begins to slip entirely to the rigid plate, which is called the gross slip The normal force about the boundary is smaller lengths of the device. The reformation criterion is based upon

than the normal force in the region of the centre. This results in a slip between the rigid plate and the elastic object reduced remarkably.

\section{CONTROLler Section OF EXOSKELETON HAND}

Controller Section is the heart of Exoskeleton Hand. The proposed exerciser, control by using a Real-Time controller. The control system of the proposed model (Fig 1.) can be divided in 3 parts: the interface running by the host computer for the instructor, a real-time controller and a visual feedback are giving by the interface to the user.

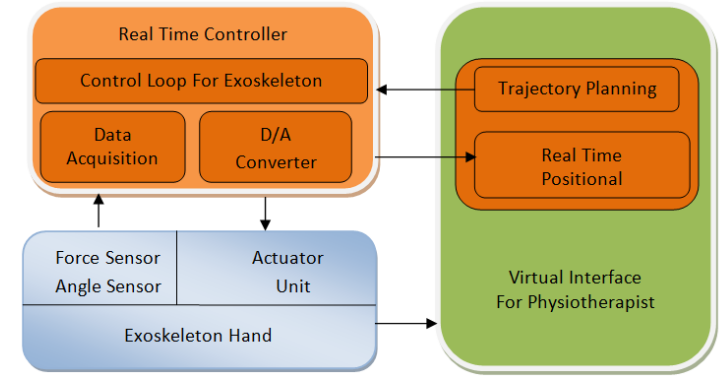

Fig. 1. Block diagram of the proposed real time controller.

The instructor interface and the user can run on different computers if required [12]. The network interfaces are connected through different parts of the system. The RTC (Real-Time Controller) from NI (National Instruments) is running the RTOS (real time operating system) and sampling all data from sensor (quadrature encoders, hall sensors, force sensors and motor currents) using data acquisition cards. All the control cycles are working well. The signal from the output channel of the PWM regulator drifts to the motors. Power calculation, no of inputs, inputs encoder and outputs are designed to control complete exoskeleton hand with 20 axes. A $1 \mathrm{KHz}$ of frequency is the smallest for PID controller. From the Lab View of the National Instrument the systems and the interfaces were developed. The interface which the advisor uses hosts by the computer displays all sensor data and also allows for required motion settings. The system even can record the movement of all joints. The motion can be played later. Any personal computer connected with the internet can run the application. Several control loops (PID) operate individually for each controlled joint. For stable motions the PID parameter of the control loop. Less precision in the position control is better than undesired overshoots or oscillations. Real time controller control 20 joints. The Exoskeleton structure rests on the hand's dorsal side and the applying forces works on that direction. The feedback force is obtained by de motors placed on a low profile power pack and transmitted to the fingers by low friction pull-cables. The finger flexi on measurement is achieved by an arrangement of flexible resistive sensors incorporated in a softly glove and custom made electromagnetic sensors combined the exoskeleton's metallic structure. The control system is shown in Fig. 2. The system is designed such that it allows integration of Hall Effect sensors for exact measurement of abduction and adduction angles of the fingers. Researchers are planning to add many features like the device always monitoring the improvement of patient. It storing the current data like force produced until pinching action occurs. This data can also help the doctor to know about the current status and trends of improvements of patients.

Arpan Bhattacharyya, Arindam Dey Sarkar and Sahadev Roy, “Active Exoskeleton Hand Exerciser Using Multisensory Feed Back,” International Journal of Advanced Engineering and Management, vol. 2, no. 2, pp. 33-36, 2017. http://ijoaem.org/00202-2 


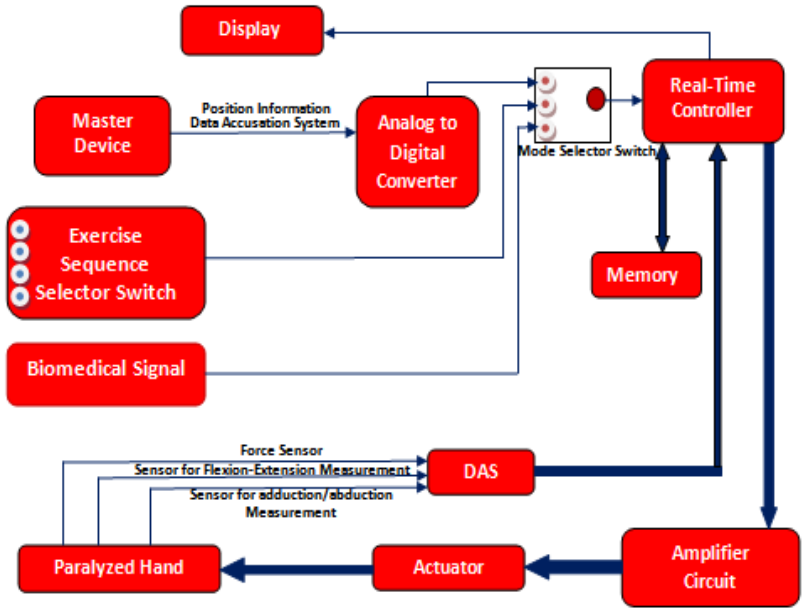

Fig. 2. Control mechanisms of the proposed system.

\section{Proposed Mechatronics Model}

By measuring the surface EMG signals from the paralyzed hand muscles, the proposed intelligent device tries to guess the stroke patient's intention and helps to complete the normal operation of the hand [13]. The system mainly based on embedded controller and a robotic hand module. The systems are available in wide range as they have lots of use in the assistance of power devices. The fingers along with the palm of the stroke patient's hand are placed on the robotic hand module by means of Velcro straps and finger rings. The DIP joint and palm area of the hand. Every hand module consists of five finger assemblies and also a platform to support palm. Each finger's joint will be actuated by using a single linear actuator and it has two DOF for every finger at the MCP and PIP jointly with help of the mechanical linkage system. PIP (proximal inter phalange) it's proximal part rotates round the centre which is virtually placed at pip joint while the distal section moves around the centre situated virtually at MCP (meta car pophalangeal) joint from totally extended to flexed position, finger assembly has sixty-five degrees and fifty-five degrees ROM (range of motion) for MCP and PIP joints respectively. In the load condition the highest contraction speed of the exoskeleton hand is just about two seconds to fully close or open the robotic hand.

After training with the device user fingers come with more firmness and can hold objects more tightly. It (the device) trained us how to performed work after the accident. The proposed rehabilitation device is specially designed for people to relearn their hand function after stroke. The concept of this device actually came from those who suffer from stroke, they want to use their hands again but the devices now available cannot help and so we begin to develop this Hand Robotic Training Device. Many of the existing devices tend to direct the hand movement and so the training is rather passive. We try to explore the probability of relying more on the user's will power, when developing the new device. This new device bring differences does to the users in the process of training that is quite observable. The previous therapy only aims to enhance the coordination of our mind, eye and hand, while the new device also includes hand strength training. This Exoskeleton Hand Robotic Training Device is mainly controlled by the muscular signals (electromyography, EMG) sent from the brain of the user. When they use their own will to open or close their hands, the device can detect the muscle signals and help open or close their hands. This device cannot move on its own, and must be driven by the intention of the user. Therefore, the user will be trained to use their brain to complete the tasks. Those who suffered from stroke are required to perform daily routines during training. They will be asked to put on the device and then move objects up and down, left and right. Each training sessions lasts for an hour. They required to practice 3 times in a week or 5 times a week while in the hospital. After successive training of 20 sessions, they will feel the difference.

\section{A. The Movement Analysis System}

First Data Glove, developed by VPL. This was a thin Lycra glove which measured the finger position by utilizing optic fibers as sensing elements. It exploits two optic fibers per finger in its standard version for the assessment of turn as well as spread of the metacarpal along with proximal inter column joints. The operating principle of data glove was calculating the intensity of light abatement as light move on all the way through the fiber optic. The cladding of fiber optic was treated at the position of the measured finger joint so that its refractive index along with that of the core material allowed abatement or contraction of light when the structure was bent. Accordingly the flexion of a joint could be measured simply by means of abatement of light intensity in the particular fibre optic. It adapted the fibre optic technology of Data Glove and linked it with new software that was used to create a tool for measurable evaluation of upper-extremity function. As the block diagram shown above containing sensing devices, which are actually helps user to learn the motion of the hand.

Master device is the basic exoskeleton structure which is harmonized with different sensors like joint positional sensor, electronics actuator control mechanism sensor, encoder etc. By these sensor present position of the different joints and links position can easily detect, which is require for controller to perform desirable task. Basically, the signal master device find out in analog form, so it goes to the ADC (Analog to Digital Converter). Exercise sequence selector switch contains some pre-programmed exercise techniques. Sequence selector having a particular series of programs which helps user to move their hand slowly. It depends on the user to select random exercises on their different mode. Biomedical signal is totally inner body signal. It comes in a form of muscles signal which a user's brain produce. It depends on the user who wants to move their hand for a several purpose.

This all signal goes to the mode selector switch where the user can select the type of exercises. It sends the output signal to the Real-Time Controller. Real-Time Controller sends the signal to the amplifier circuit for amplification where signals get amplify for the further process. It sends the signal to the actuator. Actuator contains different types of motor for the activity of the hand like dc servo motor, stepper motor, etc. Actuator detects the signal from the amplifier and move the joints of the hands accordingly that. There are three types of sensor which helps Exoskeleton hand to detect the signal .Those are Force Sensor, Sensor for Flexion-Extension Measurement, and Sensor for adduction/abduction Measurement [7]. Force sensor sense the force needs to grip or move the hand. It is actually the force or efforts the user need to put for the work to be done. Sensor for Flexion-Extension

Arpan Bhattacharyya, Arindam Dey Sarkar and Sahadev Roy, “Active Exoskeleton Hand Exerciser Using Multisensory Feed Back," International Journal of Advanced Engineering and Management, vol. 2, no. 2, pp. 33-36, 2017. http://ijoaem.org/00202-2 
Measurement is to measure the angle needs to move the hand or open the palm. It's that how much flexion or angle is needed to stretch the joints of the hand or do the exercises. Adduction/Abduction measurement sensor is same as Flexion/Extension measurement sensor. It is to measure the angle to move a body part towards the central axis of the body. Then, it goes to the DAS (Data accusation system), which sense the movement and collect the data and sends to real-time controller to control it again. The memory is here to store the controller final output for future reference and the display is to show it. In order to effectively copy the human hand for an exoskeleton application both physical and electronic design factors must be pushed to the limit. The performed design must integrate very accurate physical eminent, as well as seamless electronic integration and supporting apparatus. However, in order to further develop the human exoskeleton concept the mechanical and electronic control of the human hands must be reformed, and until this time exoskeletons will remain in their early stage of development.

\section{CONCLUSION}

The exoskeleton devices take eighty percent (80\%) of product's total weight which will not effect on human body. The device can be easily attached to the real hands. So in order to improve the results of therapy and also to reduce the rehabilitation cost of an exoskeleton hand definitely helps people. However new devices are required to improve the existing designs. These simple mechatronics device is needed to offer different types of exercise required for a patient. It can be used as new powerful light weight actuators, which are useful for both stroke therapy and impairment quantification. The device always monitors improvement of patient, while it also stores the current data like force produced during caustic action, fingertip force etc. for future reference. These data also help the doctor in identifying the current status of patient and also gives a track record of the patient's progress. The exoskeleton hand also helps the paralyzed patient to do basic tasks without external help. The development of human hand exoskeletons and robotic hands for industrial and medical applications is a very hopeful area having huge expectation. With improvement in technology, the power of actuators and decreased size of electrical stuffs, the possibility of creating very complex robotic structures in small spaces is ever increasing. However as the human hand remains the most mechanically complex and dynamic part of the human body, replicating its every function is incredibly tough. Research is going on to clearly define the drawback of forming of a dynamic control system liable for replicating the human hands movement characteristics and the actual construction of a hand exoskeleton. This clinical test concluded a significant improvement of motor abilities of patient though there was less progression in their functional skill.

\section{References}

[1] H. Kazerooni, "Exoskeletons for Human Performance Augmentation," Springer Handbook of Robotics, pp. 773-793, 2008. https://doi.org/10.1007/978-3-540-30301-5 34
[2] A. Wege and G. Hommel, "Development and Control of a Hand Exoskeleton for Rehabilitation of Hand Injuries," IEEE/RSJ International Conference on Intelligent Robots and Systems.(IROS 2005), pp. 3046-3051, 2005. https://doi.org/10.1109/iros.2005.1545506

[3] M. Fatahzadeh and M. Glick, "Stroke: Epidemiology, Classification, Risk Factors, Complications, Diagnosis, Prevention, and Medical and Dental Management.," Oral Surgery, Oral Medicine, Oral Pathology, Oral Radiology, and Endodontology, vol. 102, no. 2, pp. 180-191, 2006. https://doi.org/10.1016/j.tripleo.2005.07.031

[4] N. Van Alfen and B. G. Van Engelen, "The Clinical Spectrum of Neuralgic Amyotrophy in 246 Cases," Brain, vol. 129, no. 2, pp. 438-450, 2006. https://doi.org/10.1093/brain/awh722

[5] T. K. Tatemichi, M. Paik, E. Bagiella, D. W. Desmond, Y. Stern, M. Sano, W. A. Hauser and R. Mayeux, "Risk of Dementia After Stroke in A Hospitalized Cohort Results of A Longitudinal Study," Neurology, vol. 44, no. 10, pp. 18851885, 1994. https://doi.org/10.1212/wnl.44.10.1885

[6] S. Roy, M. S. Inamdar and S. Bhaumik, "Review of Exoskeleton Hand Exercisers for Paralyzed Patient," 2nd Research Summit on Computer, Electronics and Electrical Engineering, pp. 35-44, 2016.

[7] I. Sarakoglou, D. G. Caldwell, N. G. Tsagarakis and S. Kousidou, "Exoskeleton-Based Exercisers for the Disabilities of the Upper Arm and Hand," Rehabilitation Robotics, pp. 499-522, 2007. https://doi.org/10.5772/5177

[8] S. Lee, S. Park, M. Kim and C. W. Lee, "Design of a Force Reflecting Master Arm and Master Hand Using Pneumatic Actuators," IEEE International Conference on Robotics and Automation, vol. 3, pp. 2574-2579, 1998. https://doi.org/10.1109/robot.1998.680729

[9] C. N. Schabowsky, S. B. Godfrey, R. J. Holley and P. S. and Lum, "Development and Pilot Testing of HEXORR: Hand EXOskeleton Rehabilitation Robot," Journal of Neuro Engineering and Rehabilitation, vol. 7, no. 1, p. 36, 2010. https://doi.org/10.1186/1743-0003-7-36

[10] J. Iqbal, N. G. Tsagarakis, A. E. Fiorilla and D. G. Caldwell, "A Portable Rehabilitation Device for the Hand," Engineering in Medicine and Biology Society (EMBC), 2010 Annual International Conference of the IEEE, pp. 3694-3697, 2010. https://doi.org/10.1109/iembs.2010.5627448

[11] J. Ueda, Y. Ishida, M. Kondo and T. Ogasawara, "Development of the NAIST-Hand with Vision-Based Tactile Fingertip Sensor," Proceedings of the IEEE International Conference in Robotics and Automation, pp. 2332-2337, 2005. https://doi.org/10.1109/robot.2005.1570461

[12] R. Srivastava, N. Kumar, B. Anjali and S. N. Singh, "Grid Interactive Solar Powered Automated Bottling Plant Using Microcontroller," International Journal of Advanced Engineering and Management, vol. 2, no. 1, pp. 9-14, 2017. https://ijoaem.org/00201-7

[13] N. S. K. Ho, K. Y. Tong, X. L. Hu, K. L. Fung, X. J. Wei, W. Rong and E. A. Susanto, "An EMG-Driven Exoskeleton Hand Robotic Training Device on Chronic Stroke Subjects: Task Training System for Stroke Rehabilitation," IEEE International Conference on Rehabilitation Robotics (ICORR), pp. 1-5, 2011. https://doi.org/10.1109/icorr.2011.5975340 